\title{
Benefits of targeting both pericytes and endothelial cells in the tumor vasculature with kinase inhibitors
}

\author{
Gabriele Bergers, ${ }^{1,2}$ Steven Song, ${ }^{1,2}$ Nicole Meyer-Morse, ${ }^{3}$ Emily Bergsland, ${ }^{2}$ \\ and Douglas Hanahan ${ }^{2,3}$
}

${ }^{1}$ Department of Neurological Surgery, and Brain Tumor Research Center,

${ }^{2}$ University of California, San Francisco Comprehensive Cancer Center, and

${ }^{3}$ Department of Biochemistry and Biophysics, and Diabetes Center, University of California, San Francisco,

San Francisco, California, USA

\begin{abstract}
Functions of receptor tyrosine kinases implicated in angiogenesis were pharmacologically impaired in a mouse model of pancreatic islet cancer. An inhibitor targeting VEGFRs in endothelial cells (SU5416) is effective against early-stage angiogenic lesions, but not large, well-vascularized tumors. In contrast, a kinase inhibitor incorporating selectivity for PDGFRs (SU6668) is shown to block further growth of end-stage tumors, eliciting detachment of pericytes and disruption of tumor vascularity. Importantly, PDGFRs were expressed only in perivascular cells of this tumor type, suggesting that PDGFR $^{+}$pericytes in tumors present a complimentary target to endothelial cells for efficacious antiangiogenic therapy. Therapeutic regimes combining the two kinase inhibitors (SU5416 and SU6668) were more efficacious against all stages of islet carcinogenesis than either single agent. Combination of the VEGFR inhibitor with another distinctive kinase inhibitor targeting PDGFR activity (Gleevec) was also able to regress late-stage tumors. Thus, combinatorial targeting of receptor tyrosine kinases shows promise for treating multiple stages in tumorigenesis, most notably the often-intractable late-stage solid tumor.

This article was published online in advance of the print edition. The date of publication is available

from the JCI website, http://www.jci.org. J. Clin. Invest. 111:1287-1295 (2003). doi:10.1172/JCI200317929.
\end{abstract}

\section{Introduction}

Neovascularization is a common attribute of tumors, and a wealth of functional studies support the proposition that blood vessels are crucial for the formation, growth, and dissemination of cancer $(1,2)$. Animal models of cancer, including both traditional tumor transplants and newer genetically engineered mouse models of cancer, have helped establish the causality of angiogenesis and presented platforms for assessing antiangiogenic therapeutic strategies $(3,4)$. The latter have further revealed that the normally quiescent tissue vasculature is characteristically first activated by an "angiogenic switch" to produce new blood vessels during hyperproliferative premalignant phases of carcinogenesis, before solid tumors have formed (5-7). One such model, the RIP1Tag2 line of transgenic mice, has been particularly instructive about parameters of angiogenesis and the prospects for antiangiogenic ther-

Received for publication January 22, 2003, and accepted February 25, 2003.

Address correspondence to: Gabriele Bergers, University of California, San Francisco Comprehensive Cancer Center, San Francisco, California 94115, USA. Phone: (415) 476-6786; Fax: (415) 476-0388; E-mail: bergers@cgl.ucsf.edu.

Conflict of interest: The authors have declared that no conflict of interest exists.

Nonstandard abbreviations used: receptor tyrosine kinase inhibitor (RTKI); paraformaldehyde (PFA); FGF receptor (FGFR); platelet-endothelial cell adhesion molecule (PECAM). apy. By virtue of expressing the SV40 virus oncoproteins in the pancreatic islet $\beta$ cells, RIP1Tag2 mice develop islet carcinomas in a multistep pathway characterized by the temporal appearance of distinctive lesional stages: hyperplastic/dysplastic islets (with quiescent vasculature); angiogenic dysplastic islets; solid tumors with well-defined margins and fibrous capsules; and invasive carcinomas (8-10). The focal nature of the approximately 400 islets in the mouse pancreas and the relative synchronicity of progressive appearance of these lesions served to reveal the angiogenic switch as a discrete step in carcinogenesis (5). Furthermore, this model has afforded the design of preclinical therapeutic trials based on the distinctive stages of tumor development (3). In an assessment of four candidate angiogenesis inhibitors, differential stage-specific efficacy was observed: three agents (the protease inhibitor BB94/batimastat, endostatin, and angiostatin) performed best in treating early stage disease, both in the prevention trial targeting angiogenic switching in dysplastic lesions and in the mid-stage intervention trial aimed at blocking the expansive growth of small, solid tumors. Another inhibitor (TNP470) was effective at reducing the mass of bulky end-stage tumors in a regression trial, but it did not perform well in the early-stage prevention trial. These differential responses to antiangiogenic drugs suggested that there might be qualitative differences in the angiogenic vasculature in early and late stages or in the 
regulatory mechanisms that control induction of angiogenesis and persistence of the tumor vasculature. This concept of stage-specific efficacy has been strengthened by recent studies investigating the effects of a kinase inhibitor SU5416 (11) that selectively inhibits the VEGFRs controlling angiogenic activity of endothelial cells $(12,13)$.

Both pharmacological inhibitors and gene knockout approaches have been used to investigate the means by which the angiogenic switch is activated and sustained in this model. Key components of the switching mechanisms have proved to be a matrix protease, MMP-9, which mobilizes an angiogenic factor, VEGF-A, that in turn binds to a receptor tyrosine kinase expressed on endothelial cells, VEGFR2 $(12,14)$. Abrogation of MMP-9 by gene knockout or pharmacological inhibition reduced the frequency of angiogenic switching and impaired tumor growth (12). Furthermore, pharmacological inhibition of VEGF signaling (12) or targeted deletion of the VEGF gene (14) almost completely blocked the angiogenic switch in premalignant lesions and severely impaired growth of small tumors. The few tumors that developed in RIP1-Tag2 mice whose islets lacked VEGF, were small, avascular, and necrotic, without any features of neovascularization. These studies demonstrated the importance of VEGF-signaling for angiogenic switching, tumor formation, and initial tumor growth in this model. Remarkably, however, we have reported recently (12) that inhibition of VEGFR signaling, either indirectly with a MMPI, or directly with a VEGFRI (SU5416), was not efficacious in a regression trial against late-stage islet tumors, which continued to grow. Combination of either class of inhibitor with an antiangiogenic, "metronomic" chemotherapy regimen $(12,15,16)$ produced stable disease or modest regression of such tumors (13), encouraging the proposition that combinatorial targeted therapies might be a key to achieving late-stage efficacy with a VEGFR inhibitor. To that end we have used the RIP1Tag2 mouse model to investigate the stage specific efficacy profile of a receptor tyrosine kinase inhibitor (RTKI) with broader specificity and have assessed the benefits of combination strategies involving distinctive RTKIs. The studies reported below encourage multiplex receptor-targeting strategies and have in particular highlighted the potential significance of PDGFR signaling in tumor-associated pericytes, thereby implicating this cell type as a functionally important component of the tumor vasculature and a new target for antiangiogenic therapy.

\section{Methods}

Drug treatment of transgenic mice. The mice in these studies were males and females of the RIP1Tag2 transgenic mouse lineage that were bred 45 generations into the $\mathrm{C} 57 \mathrm{Bl} / 6 \mathrm{~J}$ background. Animals were treated from 5 to 10.5 weeks of age in the prevention trial, from 10 to 13.5 weeks in the intervention trial, and from 12 to 16 weeks in the regression trial. All control mice received subcutaneous or oral saline injections. SU6668 and SU5416
(Sugen Inc., South San Francisco, California, USA) were provided in vehicle formulations. As single agents, 200 $\mathrm{mg} / \mathrm{kg}$ of SU6668 was administered orally every day, and $100 \mathrm{mg} / \mathrm{kg}$ SU5416 was inoculated subcutaneously twice a week; if combined with other drugs, the SU5416 dose had to be reduced to $50-75 \mathrm{mg} / \mathrm{kg}$. Studies were limited by toxicities (weight loss, lung hemorrhages) associated with SU5416, predominantly in end-stage mice (older than 14 weeks), which appear to relate to the chemistry of SU5416 rather than its mechanism of action. We have not, for example, observed such toxic side effects in trials with another VEGFR inhibitor (O. Casanovas and D. Hanahan, unpublished observations). Gleevec/STI57 (50 mg/kg; Novartis Pharma AG, Basel, Switzerland) was administered orally twice a day (17). All trials were repeated up to three times. Mice were maintained in accordance with the University of California, San Francisco (UCSF) institutional guidelines governing the care of laboratory mice, and euthanized after the respective treatment period or when tumor burden and/or side effects obligated their removal from study.

Assessment of the angiogenic islets and tumor burden. In the prevention trial, angiogenic islets were isolated by retrograde perfusion with collagenase solution and counted. Angiogenic islets were identified as those that exhibited a reddish patch or patches (caused by hemorrhaging) in a white nodular background (18). In the intervention and regression trials, animals were euthanized at the end of the respective trial and tumors microdissected from freshly excised pancreata. Tumor volume (cubic millimeters) was measured by using a caliper, applying the formula [volume $=0.52 \times(\text { width })^{2} \times($ length $)$ ] for approximating the volume of a spheroid. Tumor burden per mouse was calculated by accumulating the tumor volume of every mouse.

Visualization of the vasculature. To visualize blood vessels in tumors and normal tissue, mice were first anesthetized and injected intravenously with $0.05 \mathrm{mg}$ FITC-labeled tomato lectin (Lycopersicon esculentum; Vector Laboratories, Burlingame, California, USA), then the heart was perfused with $4 \%$ paraformaldehyde (PFA). Pancreata were frozen in OCT medium and sectioned at $50 \mu \mathrm{m}$.

Immunobistochemical analysis. Mice were anesthetized, hearts perfused with PFA, and pancreata collected, frozen in OCT medium, and $15-\mu \mathrm{m}$ sections prepared. Pericytes were identified with a mouse anti-human desmin Ab (1:3,000; DAKO Corp., Carpinteria, California, USA), a marker of mature pericytes, and endothelial cells were detected with a rat anti-mouse CD31 Ab (1:100, BD PharMingen, San Diego, California, USA). To reveal the $\mathrm{Ab}$ reactions, sections were then incubated with either a CY3-labeled goat anti-mouse IgG Ab (Jackson ImmunoResearch Laboratories Inc., West Grove, Pennsylvania, USA), or a FITC-labeled goat anti-mouse IgG Ab (Jackson ImmunoResearch Laboratories Inc.), or a rhodamine-labeled goat anti-rat IgG Ab (Jackson ImmunoResearch Laboratories Inc.). To visualize endothelial and perivascular cells, sections were either simultaneously stained with a CD31 and desmin 
$\mathrm{Ab}$, or immunohistochemistry was performed with the desmin $\mathrm{Ab}$ on cryosections of mice whose vascular system had been perfused with lectin-FITC before euthanasia. PDGFR- $\beta^{+}$cells were deleted with a ratautoimmune PDGFR- $\beta$ antibody from eBioscience (San Diego, California, USA).

FACS analysis. Mice were sacrificed and tumors excised from the pancreas and minced with a razor blade on ice in $1 \times$ PBS. The minced tumor fragments were then digested at $37^{\circ} \mathrm{C}$ for 13 min with a collagenase mix containing $0.2 \mathrm{~g}$ BSA (Sigma Aldrich, St. Louis, Missouri, USA), $0.05 \mathrm{~g}$ collagenase II, $0.05 \mathrm{~g}$ collagenase IV, 0.02g DNase I (all from Worthington Biochemical Corp., Lakewood, New Jersey, USA), and passed through a $70-\mu \mathrm{m}$ cell strainer. The cells were washed, the red blood cells lysed with PharM Lyse (BD PharMingen) for $15 \mathrm{sec}$, and then washed again. The cell pellets were resuspended in FACS buffer $(1 \times$ PBS plus 1\% BSA), preblocked with an Fc block (CD16/CD32; BD PharMingen), and then incubated with primary $\mathrm{Ab}$ on ice: CD31-PE, 1:100; Ly-6G, 1:60 (recognizing the GR-1 antigen on granulocytes), from BD PharMingen; CD11b, 1:40 (recognizing the Mac-1 antigen on macrophages) and other immune cell types from BD PharMingen. The cells were washed and ViaProbe was added as a cell death indicator (BD PharMingen). The cells were then sorted on a FACSVantage SE flow cytometer using the Cell Quest Pro software version 4 from Becton Dickinson Immunocytometry Systems (Franklin Lakes, New Jersey, USA). The FL2 gate identified the $\mathrm{CD} 31^{+}$cells, the FL1 gate identified the $\mathrm{Gr}-1^{+} / \mathrm{Mac}-1^{+}$cells, and the FL3 gate excluded the dead cells.

$R N A$ isolation and RT-PCR analysis. FACS-sorted cells were collected in a cell lysis solution from QIAGEN Inc. (Valencia, California, USA) containing $\beta$-mercaptoethanol. RNA was isolated following RNeasy Mini Kit protocols (QIAGEN Inc.) and transcribed into single-stranded cDNA using Superscript II RNase $\mathrm{H}^{-}$ Reverse Transcriptase (Invitrogen Corp., Carlsbad, California, USA). RT-PCR analysis was performed using custom primers (QIAGEN Inc.) for PDGF-A, -B, -C, -D, PDGFR- $\alpha$ and $-\beta$, desmin, and smooth-muscle actin, and for L19 as internal control.

\section{Results}

Two RTKIs have distinctive efficacy profiles. Previously, we evaluated the VEGFR inhibitor SU5416 $(11,19)$ in the three distinctive therapeutic trials in RIPTag2 mice (12, 13); these data are illustrated here to facilitate evaluation of the comparative and combination trials involving this and other RTKI. In the regression trial, which treats end-stage mice having bulky disease, thus being analogous to the typical phase- 3 clinical trial of investigational anticancer drugs (Figure 1), treatment with SU5416 produced a modest increase in life span (to a defined endpoint 2.5 weeks after sham-treated mice were euthanized due to incipient death from tumor burden), concomitant with a lower rate of tumor growth, but this drug was not capable of regressing tumor mass or producing stable disease (Figure 1; RT). As such, we found the SU5416 efficacy profile to be similar to that of endostatin, angiostatin, and the MMP inhibitors BB94 and BAY-129566 $(3,12,13)$.

Given that SU5416 was very efficacious against earlier-stage disease (Figure 1), phenocopying the VEGF-A gene knockout in its impairment of angiogenic switching and tumor growth, we reasoned that other regulatory molecules might become involved in controlling angiogenesis and maintaining of the tumor vasculature in well-established solid tumors. We therefore evaluated another RTKI (SU6668) with somewhat broader selectivity in the three distinctive preclinical trials in the Rip1Tag2 model.

SU6668, a small molecule kinase inhibitor with demonstrable antiangiogenic activity (20-22), inhibits phosphorylation and signal transduction of PDGFRs, VEGFRs, and FGF receptors (FGFRs). While SU6668 has significantly higher biochemical activity against PDGFR- $\alpha$ and $-\beta\left(K_{\mathrm{I}}=0.0008 \mu \mathrm{M}\right)$ than VEGFR-2 and FGFR-1 $\left(K_{\mathrm{I}}=2.1 \mu \mathrm{M}\right.$ and $1.2 \mu \mathrm{M}$, respectively) (21), cellbased assays reveal functionally appreciable inhibitory
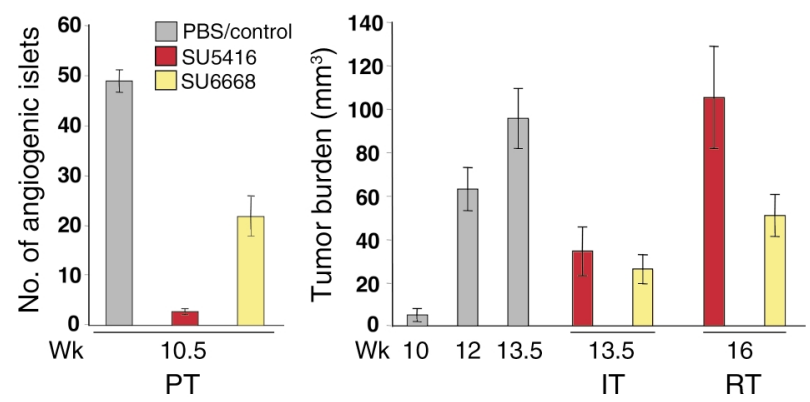

\section{Figure 1}

Different stage-specific efficacy profiles for the VEGFR inhibitor SU5416 and the PDGF (+VEGF/FGF) receptor inhibitor SU6668 in the three distinct stages of pancreatic islet carcinogenesis in RIP1Tag2 transgenic mice. Mice were either treated with SU5416 or SU6668 as described in Methods. The average number of angiogenic islets \pm SEM at 10.5 weeks in control and treated mice, the average tumor burden \pm SEM in PBS/vehicle-treated mice (at 10, 12, 13.5 weeks), and SU5416- and SU6668-treated mice (at 13.5 and 16 weeks) are shown. The prevention trial (PT) started at 5 weeks, when mice harbor hyperplastic/dysplastic islets, and ended at 10.5 weeks, when the first small tumors appear. Islets that have switched on angiogenesis are scored by their reddish color (resulting from microhemorrhage and leakiness associated with VEGF-induced angiogenesis). In the intervention trial (IT), mice with a small tumor burden (10 weeks) are treated until the end stage (13.5 weeks), while in the regression trial (RT), 12-week-old mice with substantial tumor burden and a life expectancy of less than 2 weeks are treated until 16 weeks, when control mice are already dead. Statistical analysis was performed with a two-tailed, unpaired Mann-Whitney test comparing experimental groups to PBS-injected control mice. Tumor burdens of experimental groups in the Regression Trial were compared to that of 12-week-old Rip1Tag2 mice. Cohorts of 6-21 animals were used. $P$ values less than 0.1 are considered statistically significant. $P$ values of SU5416 PT $=2.26 \times 10^{-5}$, SU6668 PT $=0.0002$, SU5416 IT $=0.0009$, SU6668 $\mathrm{IT}=0.0001, \mathrm{SU} 5416 \mathrm{RT}=0.1827$, and SU6668 RT $=0.3228$. 

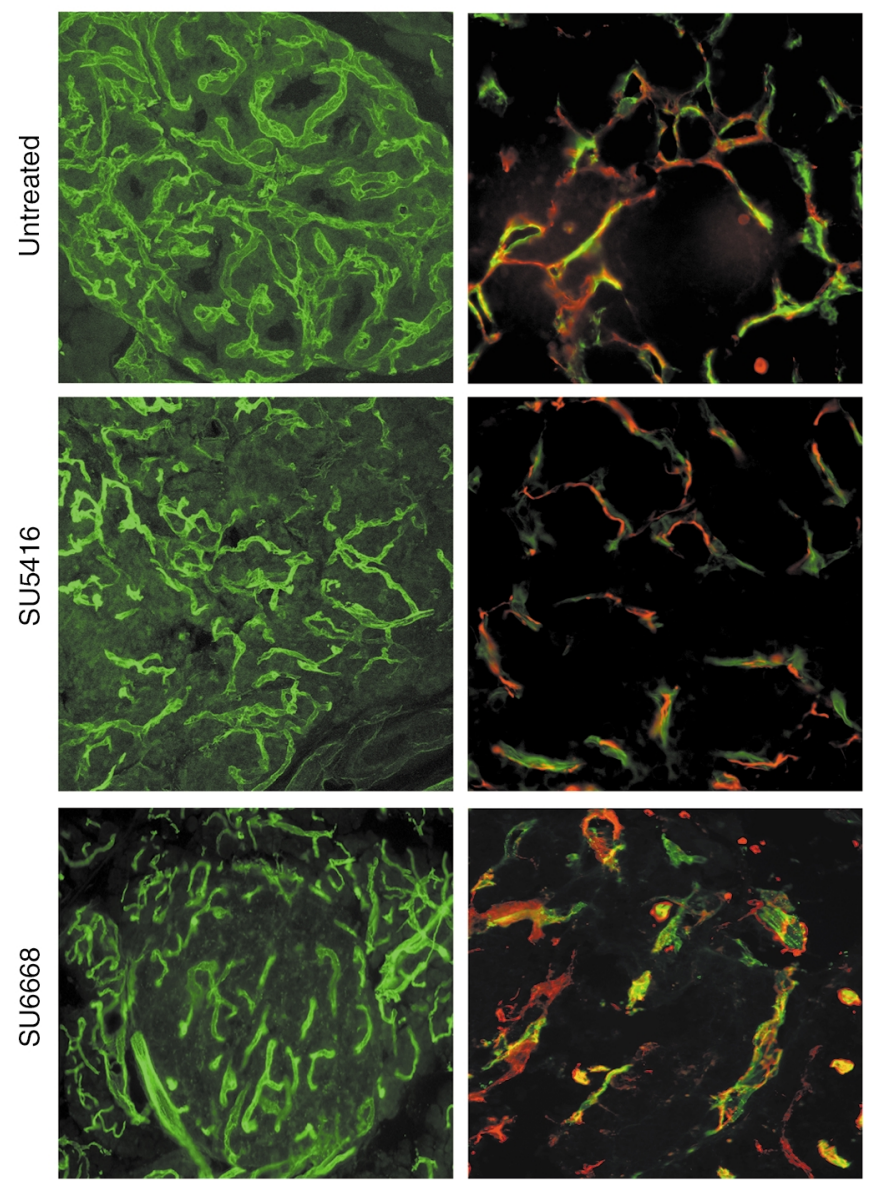

\section{Figure 2}

Comparison of vascular morphology (left panels) and association of endothelial cells and perivascular cells (right panels) in treated versus control tumors. Tumor-bearing pancreata were taken from end-stage 13.5-week-old control Rip1Tag2 mice and from 16-week-old Rip1Tag2 mice treated with SU5416 or SU6668 (Regression Trial). To visualize the functional blood vessels in tumors, mice were first anesthetized and injected intravenously with $0.05 \mathrm{mg}$ FITC-labeled tomato lectin (Lycopersicon esculentum) and then heart perfused with 4\% PFA. Pancreata were frozen in OCT medium and sectioned at $50 \mu \mathrm{m}$. To visualize endothelial cells (green in right panels) and pericytes (red in right panels) by immunohistochemical analysis, mice were anesthetized, heart perfused with PFA, and pancreata collected, frozen in OCT medium, and $15-\mu \mathrm{m}$ sections prepared. Endothelial cells were detected with FITC-labeled lectin; pericytes were identified with CY3-labeled anti-desmin $(1: 3,000)$, a marker of mature pericytes.

investigate the expression of PDGF ligands and receptors in the islet tumors, given that SU6668 potently inhibits these receptors.

To investigate the underlying mechanisms of these stage-specific effects, we first assessed the vascular morphology in the treated versus control tumors from the regression trials of these two drugs. Perfusion of the circulatory system with a fluorescent lectin was used to assess the functional tumor vasculature, revealing (Figure 2; left panel) demonstrable decrease in vascularity of SU6668-treated tumors and modest reduction in tumors treated with SU5416. We further assessed the vascular morphology by immunostaining tissue sections with $\mathrm{Ab}$ 's

activity against both VEGFR-21 and PDGFR (21, 23, 24). By contrast, SU5416 is predominantly active against VEGFR-2 $\left(K_{\mathrm{I}}=0.04 \mu \mathrm{M}\right)$, with minimal activity against PDGFR or FGFR $(11,25)$.

The trials with SU6668 produced a different efficacy profile from that of SU5416. Thus, SU6668 was less effective at blocking angiogenic switching in the prevention trial and was similar at impairing tumor growth in an intervention trial (Figure 1). Surprisingly, this agent proved much more effective than SU5416 in the regression trial, producing a condition of stable disease, in that tumor burden at the culmination (a defined end point after 4 weeks of treatment) was similar to that at the 12 -week-old starting point. How can these differences be explained? We suspect that the relatively poor activity of SU6668 at blocking angiogenic switching (Figure 1; PT) reflects its lower $K_{\mathrm{I}}$ against VEGFR; by contrast, SU5416 produced similar reductions in angiogenic switching to the gene knockout of $V E G F-A$ (14), indicative of the singular importance of VEGF signaling at this early stage. As for its significantly better effects on well-established tumors, we hypothesized that the broader specificity of SU6668, in particular against PDGFR, might underlay its benefits. We sought, therefore, to compare the histological effects of treatment with these two agents that might reflect distinctive functional effects and to recognizing two vascular markers: CD31 (PECAM), a cell adhesion molecule expressed on endothelial (and hematopoietic) cells, and desmin, a marker of mature periendothelial support cells (pericytes) $(26,27)$. We were motivated to investigate pericytes both by a report (28) and our unpublished data that pericytes were abundant in tumors of the RIP1Tag2 model. A typical intimate association was seen between endothelial cells and pericytes in the untreated tumors, as well as in the SU5416-treated tumors (Figure 2; right panel). By contrast, SU6668-treated tumors showed marked disruption of pericyte-endothelial cell association. Pericytes had become separated from the endothelial cells, and the blood vessels were enlarged and distorted (Figure 2). Thus, SU6668 affected the tumor vasculature differently than SU5416: not only was the vascularity of the tumors reduced, but the integrity of the association of endothelial cells and pericytes was markedly perturbed. Collectively, the results led us to suspect that the distinctive effects of SU6668 against solid tumors resulted from its targeting of pericytes; this was further supported by a report suggesting that SU6668 could reduce the number pericytes in a tumor transplant model (29). Further rationale for this hypothesis came from the knowledge that pericytes express PDGFRs during vessel formation in the developing embryo and that functional disruption of PDGFR- $\beta$ or its ligand 
PDGF-B leads to a lack of pericytes, causing severe vascular defects and embryonic lethality in late gestation (30-32). Such considerations raised a question: were PDGFRs expressed in pericytes or other cell types in the islet tumors, and if so were PDGF ligands expressed?

Expression in tumor vasculature of PDGF ligands and PDGFRs. To assess the expression of PDGF ligands and PDGFRs, RNA was isolated from whole tumors and analyzed by RT-PCR, revealing expression of the PDGF-A, -B, and -D ligands, as well as both PDGFR- $\alpha$ and $-\beta$ (Figure $3 a$ ). To identify the cell type that expressed PDGF ligands and receptors, we developed a protocol for fractionating primary tumors into constituent cell types by flow cytometry. Endothelial cells were sorted as $\mathrm{CD}^{2} 1^{+}, \mathrm{Gr}^{-}$, Mac1-; inflammatory cells were collected as $\mathrm{Gr}^{+}$, $\mathrm{Mac1}^{+}$(not shown); and tumor cells were gated by size and collected as unlabeled with these three Ab's. RNA was collected from unsorted and sorted populations and analyzed by RT-PCR. As illustrated in Figure 3a, the endothelial cells were found to exclusively express the genes for PDGF ligands A, B, and D. None of the sorted cell populations, tumor cells, inflammatory cells (not shown), or endothelial cells, expressed the two PDGFR genes, despite demonstrable expression in whole-tumor RNA. We therefore stained tumor tissue sections with Ab's specific for PDGFR- $\beta$ to ask whether the receptor was expressed, and if so, in which cell type. The tissue sections were costained with anti-CD31/PECAM to mark the endothelial cells. The data shown in Figure $3 \mathrm{~b}$ clearly reveal expression of PDGFR- $\beta$ in perivascular cells that are in close contact with endothelial cells, but not in the endothelial cells or tumor cells, consistent with the analysis of the sorted cell types. (PDGFR- $\alpha$ expression is very low in the pancreatic tumors, and the mouse protein could not be detected with available Ab's in tissue sections.) We went on to collect the PDGFR ${ }^{+}$cells by FACS using an anti-PDGFR- $\beta$ Ab; this cell population proved to have a small size that was gated out in the unlabeled tumor cell fraction shown in Figure 3a and furthermore expressed known markers of pericytes (e.g., desmin and smooth-muscle actin [SMA], as shown in Figure 3c) consistent with identification of the PDGFR- $\beta^{+}$cells in these tumors as a class of pericyte. The data indicate that PDGF ligands are expressed in the tumor endothelial cells and that PDGFR- $\beta$ is expressed in cells associated with the angiogenic vasculature that morphologically score as perivascular cells (pericytes). The data are consistent with the hypothesis that SU6668 is targeting PDGFR ${ }^{+}$ pericytes, causing their dissociation from the tumor vasculature, leading to vascular dysfunction. It is formally possible that the VEGFR activity of SU6668 is contributing to the observed effects on tumor growth and tumor vascularity. The VEGFR selective inhibitor SU5416 did not induce dissociation of pericytes from tumor blood vessels (Figure 2), however, suggesting that SU6668's modest activity against the VEGFRs is not the primary basis for its effect on tumor pericytes.

We cannot at present exclude possible contributions to the observed effects of SU6668's inhibition of FGFRs, given that there is evidence supporting involvement of FGF signaling in this model (33). Data to be presented below, however, suggest that the primary activity of SU6668 in this model of pancreatic islet carcinogenesis involves its inhibition of PDGFR signaling.

Improved efficacy by combining kinase inhibitors with distinct specificity. SU5416 was most efficacious at blocking initial angiogenic switching and similarly as effective as SU6668 at repressing growth of small, nascent, solid tumors, whereas SU6668 was more effective against end-stage bulky disease (Figure 1). Therefore, we reasoned that combining the two kinase inhibitors might improve efficacy, given their distinctive efficacy profiles and target selectiveness. To investigate this hypothesis, combination trials were performed. The data are provocative. The combination of SU5416 and SU6668 improved efficacy in each of the three trials,

\section{a}

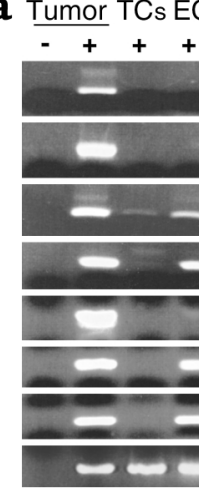

PT
PDGFR- $\alpha$
PDGF-A
PDGF-B
PDGF-C
PDGF-D
VEGFR2
19 (control)

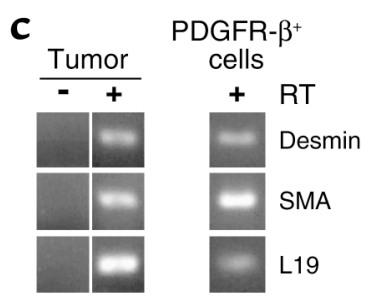

b

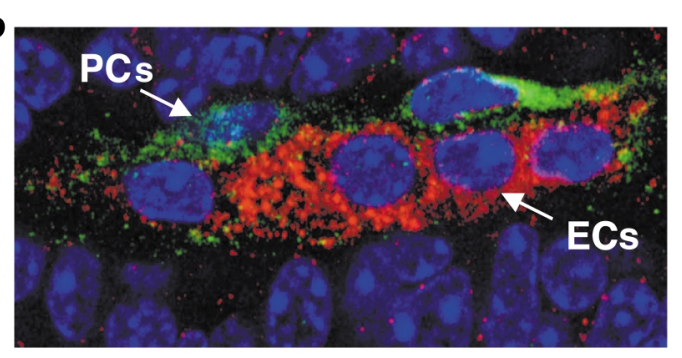

Figure 3

Identification of the cell types expressing PDGF ligands and receptors in pancreatic islet carcinomas. (a) Primary tumors were fractioned into constituent cell types by flow cytometry. RNA was isolated from unsorted and sorted populations and analyzed by RT-PCR. Pancreatic tumors of end-stage Rip1Tag2 mice were excised and enzymatically dispersed with collagenase into single cells. The cell suspension was incubated with Ab's for CD31 and Gr1 and Mac1. Endothelial cells were collected by FACS as a $\mathrm{CD} 31^{+}, \mathrm{Gr} 1^{-}$, Mac1- population, whereas tumor cells were gated by size and collected as unlabeled with these three Ab's. Inflammatory cells were collected as $\mathrm{Gr}^{+}$, $\mathrm{Mac}^{+}$; these cells did not express PDGF ligands or receptors (not shown). (b) Tumor sections (prepared as in Figure 2) were costained with anti-PDGFR- $\beta$-FITC $(1: 200)$ and anti-CD31-rhodamine to reveal PDGFR- $\beta$-expressing cells in green and endothelial cells in red. (c) PDGFR- $\beta^{+}$cells from tumors were isolated by FACS (PDGFR- $\beta$ Ab, $1: 50)$, RNA isolated, and analyzed by RT-PCR for pericyte markers. ECs, endothelial cells; TCs, tumor cells; PCs, perivascular cells. 

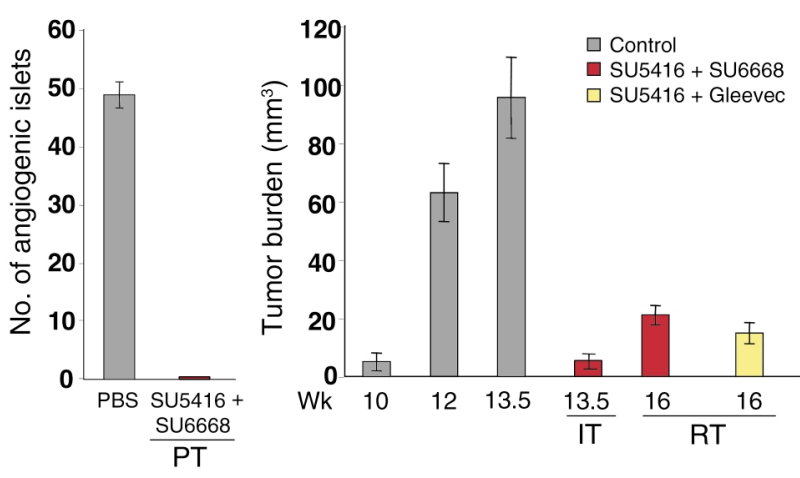

\section{Figure 4}

Improved efficacy at all stages of islet carcinogenesis produced by combining the VEGFR-inhibitor SU5416 with SU6668 or Gleevec, two drugs that inhibit PDGFR signaling. Mice were injected subcutaneously with $50-75 \mathrm{mg} / \mathrm{kg}$ SU5416 twice a week and in addition received either daily oral administration of $200 \mathrm{mg} / \mathrm{kg}$ SU6668 or twice daily dosing of $50 \mathrm{mg} / \mathrm{kg}$ Gleevec (STI571). (The dosage of SU5416 had to be reduced from that used in the single-agent trials shown in Figure 1 due to SU5416-specific toxic side effects.) The average number of angiogenic islets \pm SEM at 10.5 weeks in control and treated mice are shown in the prevention trial. The average tumor burden \pm SEM of PBS/vehicle-treated mice is indicated at 10,12 , and 13.5 weeks, for comparison with SU5416 + SU6668-treated mice at 13.5 and 16 weeks, and with SU5416 + Gleevec-treated mice at 16 weeks. Tumor burdens were assessed as described in Methods. Statistical analysis was performed with a two-tailed, unpaired MannWhitney test comparing experimental groups with PBS-injected control mice. Tumor burdens of experimental groups in the regression trial were compared with that of 12-week-old Rip1Tag2 mice. Cohorts of 6-21 animals were used. $P$ values less than 0.1 are considered statistically significant. ( $P$ values of SU5416 + SU6668 PT $=0.0002$, SU5416 + SU6668 IT $=0.0008$, SU5416 + SU6668 RT $=0.0003$, and SU5416+ Gleevec RT $=0.0007$.

variously targeting angiogenic dysplasias, small tumors, or end-stage tumors (Figure 4). Thus, the combination was better than either of the single agents at all stages of carcinogenesis, producing a broad efficacy profile. Mice treated with the combination did not develop morphologically identifiable angiogenic islets in the prevention trial, in contrast with the control mice. Histological analysis revealed that treated islets did not have hemorrhages and appeared more benign, like early hyperplastic islets in which the cells had a larger cytoplasm-to-nucleus ratio (Figure 5, a and b). Most exciting was the clear and convincing reduction in tumor mass seen in the regression trial. Not only did the combination produce severe reductions in tumor mass (Figures 4 and 5, c and d), but it also elicited evident necrosis and limited the characteristic microhemorrhaging that produces blood-filled tumors (Figure 5, e and f). The blood vessel network regressed and predominantly disappeared in larger tumors, leaving the perivascular cells stretched out and distorted (Figure 5, g and h). Concomitantly, apoptosis was increased 6.7-fold (Figure 5, $i$ and j) in the combination therapy, whereas single treatment with SU5416 or SU6668 increased apoptosis 2.7 - and 3.5-fold, respectively. The data presented above suggested that these dramatic effects are the result of simultaneous targeting of VEGFRs in endothelial cells and PDGFRs in perivascular cells. The modest inhibitory profile of SU6668 against FGFRs and its demonstrable VEGFR activity, however, cannot absolutely exclude other interpretations. Therefore, we tested another tyrosine kinase inhibitor, Gleevec (STI571), which has high activity against three kinases: Bcr/Abl, c-Kit, and PDGFR (17, 34, 35). Among known kinases tested, Gleevec overlaps with SU6668 only in its inhibition of PDGFR. We performed a regression trial with Gleevec, treating near end-stage mice with Gleevec alone or in combination with SU5416. Gleevec, which has very poor pharmacokinetic properties in mice (R. van Etten, Harvard Medical School, Boston, Massachusetts, USA, personal communication; see ref. 34), was not effectual as a single agent (not shown). Nevertheless, the combination of Gleevec and SU5416 produced significant reductions in tumor burden (Figure 4) comparable to SU6668 plus SU5416 treatment. Furthermore, the Gleevec combination produced similar morphological disruptions in tumors (increased apoptosis, decreased abundance and detachment of pericytes, and reduced vascularity; Figure 51). Interestingly, the degree of vascular disruption varied among independent tumors, apparently as a function of the size of tumors, being most apparent in larger tumors. Importantly, neither of the combinatorial treatments caused dissociation of perivascular cells from blood vessels of normal tissue (lung, liver; data not shown) or otherwise disrupted normal tissue vasculature, including that of the immediately adjacent exocrine pancreas to the islet tumors (shown for Gleevec plus SU5416 in Figure 5k), indicating that the tumor vasculature is differentially sensitive to these kinase inhibitors.

The dramatic effect both kinase inhibitor combinations had on tumors in a month-long trial raised the question of whether they could improve survival over a longer time period. We sought to treat end-stage mice in a multimonth trial, but found that SU5416 was poorly tolerated by older mice, requiring most mice to be removed from study (see Methods). These side effects precluded statistically significant survival studies. Nevertheless, a few mice were able to stay in trial for 2 months with SU6668 plus SU5416 in the regression trial (i.e., 6 weeks past the incipient death and obligate euthanasia of the untreated controls); these mice still had a smaller tumor burden than mice at the starting point of the trial (12 weeks) (data not shown), indicating that the combination therapies were not only regressing well-established tumors in end-stage mice, but also limiting subsequent regrowth of these tumors or other new tumors forming from the abundant angiogenic progenitors characteristic of this challenging multifocal model of carcinogenesis. We hope to address long-term survival in future studies using less toxic VEGFR inhibitors in conjunction with Gleevec and SU6668, as well as other PDGFR inhibitors. 


\section{Discussion}

In the course of investigating the efficacy of RTKIs in a mouse model of multistage carcinogenesis, we have made an unexpected observation that may have important implications for therapeutic strategies targeting angiogenesis and the tumor vasculature for the treatment of human cancers. The data support the proposition that perivascular cells associated with the tumor vasculature expressing the PDGFR- $\beta$ are functionally important for maintenance of tumor blood vessels, adding another constituent cell type in tumors to the list of anticancer targets (Figure 6). Association of $\mathrm{PDGFR}^{+}$perivascular cells with the tumor endothelial cells is ostensibly maintained by the expression of PDGF ligands in the endothelial cells, establishing a paracrine homeostatic signaling circuit analogous to the situation during embryonic development of certain tissue vascular beds $(27,31,36)$. Notably, neither PDGF ligands or PDGFRs are expressed by the tumor cells in this model of pancreatic islet carcinogenesis, unlike many of the tumor transplant models studied with the kinase inhibitors under consideration (20,29). The lack of tumor cell expression points to the importance of PDGF signaling in perivascular cells for sustaining the tumor vasculature via association with endothelial cells.

There are several reasons to suspect that the actions and interactions of pericytes and endothelial cells in these prototypical tumors are qualitatively different from that in normal tissues. First, the SU6668 single treatment, as well as the combined treatment of SU5416 plus SU6668 or Gleevec, which disrupts the association of pericytes and reduces the vascularity in tumors, has no such effect in normal tissues of the treated mice. This is in agreement with the finding that perturbation of PDGF signaling in the developing retina elicits detachment of pericytes on immature vessels, but not mature vessels, indicating the pericyteendothelial interactions in newly formed vessels is critically dependent on PDGF $(24,32,37)$. Second, blockage of VEGFR also disrupts the tumor vasculature but
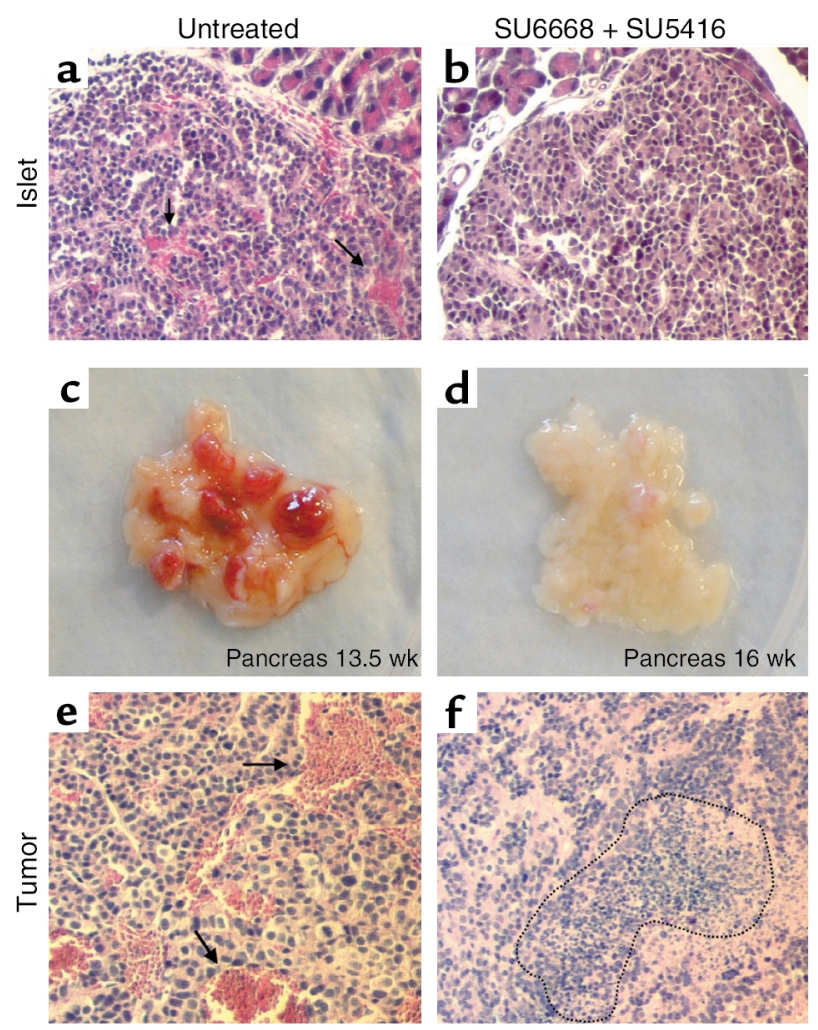
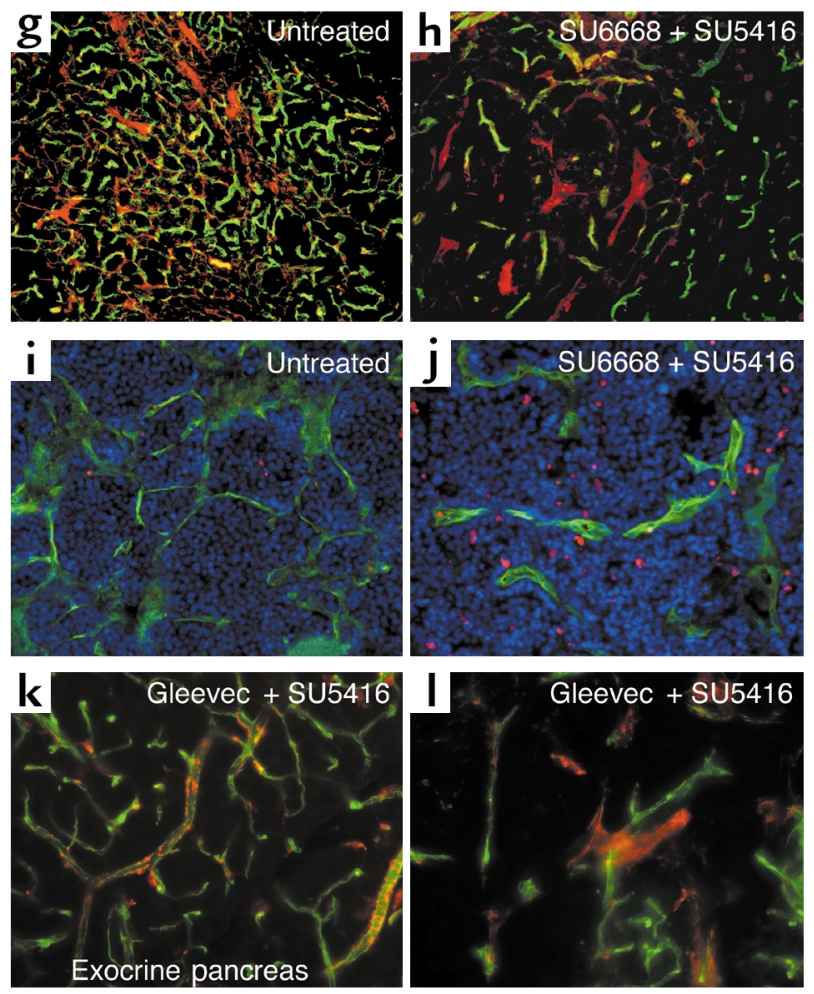

\section{Figure 5}

Effects of the combined therapy using SU5416 + SU6668 or SU5416 + Gleevec. Hematoxylin and eosin staining of islets from untreated (a) and SU6668 + SU5416-treated transgenic mice (b) at 10.5 weeks in a prevention trial. Gross pathology of dissected pancreata from untreated (c) and SU5416 + SU6668-treated mice (d) in a 4-week regression trial targeting late-stage disease. Hematoxylin and eosin staining of tumors from untreated (e) and SU5416 + SU6668-treated mice (f). Arrows indicate hemorrhage formation, and dotted area confines necrotic region. Comparison of the functional vasculature in control (g) and SU5416 + SU6668-treated mice (h) from a regression trial. Mice were injected intravenously with FITC-labeled tomato lectin (Lycopersicon esculentum) to stain blood vessels in green, and then heart perfused with 4\% PFA, followed by immunohistochemical staining with Cy3-labeled desmin Ab to label desmin-expressing perivascular cells in red. Apoptotic cells in tumors of control (i) and SU6668 + SU5416-treated mice (j) were detected by TUNEL staining with fluorescent visualization (red), and the vasculature was revealed as above by intravenous FITC-lectin perfusion before sacrifice. Mice were treated with SU5416 + Gleevec in the regression trial, and blood vessels and perivascular cells of exocrine pancreas (k) and adjacent islet tumors (I) were visualized with FITC-lectin and a Cy3-labeled desmin Ab. 


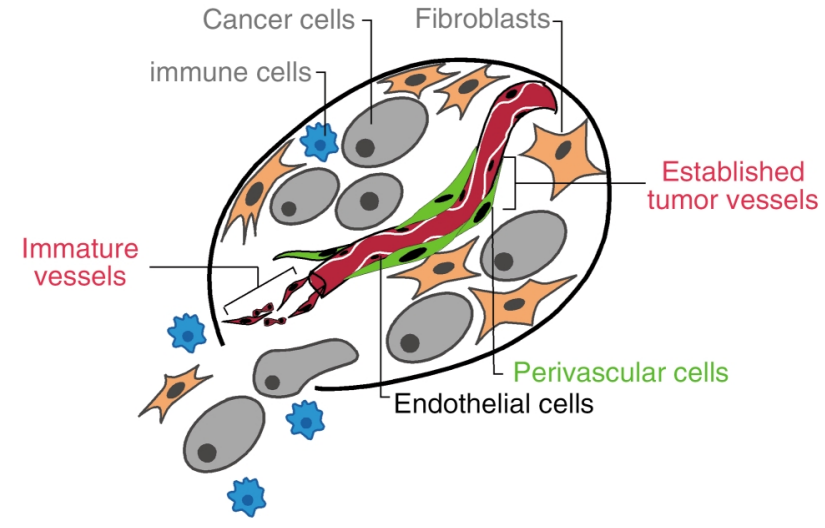

\section{Figure 6}

Two distinctive vascular cell types in tumors present complementary targets for anticancer drugs. The results presented herein suggest that the conceptual notion of tumors as aberrant organs composed of both cancer cells and conscripted normal cell types, all making functional contributions to tumor phenotypes (43), be expanded both to include PDGFR- $\beta^{+}$pericytes and to recognize that tumors can have vasculature that is either immature or mature, with different responses to angiogenesis inhibitors. Combined therapy efficacy against otherwise intractable late-stage islet carcinomas is observed when VEGFRs on endothelial cells and PDGFRs on perivascular cells are targeted together. There is reason to envision that when the other constituent cell types, in particular the overt cancer cells, are also targeted, long-term therapeutic benefit can be achieved.

not normal tissue vessels, again revealing different sensitivity. Its effect on tumors was markedly different, however: treatment with the VEGFR-selective inhibitor SU5416 had no impact on pericyte-endothelial association in islet tumors (see Figure 2) despite its clear impairment of angiogenesis. Taken together, these observations suggest that the endothelial cells and pericytes in tumors are abnormal in their regulation and likely their functionality, providing a rationale for the "therapeutic window" we observe in the ability of these agents to selectively disrupt tumor vasculature and effect tumor regression. The basis of the apparent differences in normal and tumor pericytes and endothelial cells and in their paracrine homeostatic signaling deserves future investigation. It is of interest that PDGF signaling recently has been implicated in maintenance of interstitial pressure in tumors, in that inhibitors of PDGFR (including Gleevec) reduced pressure in subcutaneous transplant tumors (38); perhaps impaired pericyte interactions with the tumor vasculature is contributing to this effect.

The use of distinctive preclinical trials targeting different stages in the development of islet carcinomas in the RIP1Tag2 mouse model has revealed a stage-specific efficacy for the VEGFR inhibitor SU5416, which is similar to that of protease inhibitor BB-94, which targets the VEGF-A-activating protease MMP-9 $(3,12,13)$. Both investigational drugs were effective in treating premalignant lesions (angiogenic dysplasias) and small tumors, but neither was able to produce responses against late-stage tumors. The lack of efficacy against large tumors is congruent with the failure of three MMPIs in phase- 3 clinical trials against late-stage human cancers $(39,40)$ and predicts the same for VEGFR inhibitors: notably, SU5416 was recently withdrawn from phase-3 clinical trials against late-stage cancers, perhaps reflecting a similar lack of efficacy (http://www.sugen.com/webpage_templates/sec.php3?p age_name=pr_1013191807\&press_release $=18 y e a r=$ 2002). By contrast, SU6668 had a distinctive efficacy profile in the stage-specific trials in the RIPTag2 model: it was less effective in the prevention trial targeting angiogenic dysplasias, but was much better in the regression trial, producing a condition of "stable disease." Remarkably, the combination of the two RTKIs produced clear and convincing responses in all three trials, which in every case was better than either single agent alone. The synergy supports the arguments above that these agents are targeting different signaling circuits and, indeed, distinct vascular cell types in tumors. The proposition that SU6668 is predominantly an inhibitor of PDGFR signaling is supported by the combinatorial trials using SU5416 plus Gleevec, which inhibits PDGFR along with $\mathrm{Bcr} / \mathrm{Abl}$ and c-Kit, but not FGFR or VEGFR. Thus, SU6668 and Gleevec share activity only against PDGFR, among the known kinases tested. Certainly highly specific inhibitors that pharmacologically knock out only PDGFR signaling will be necessary to unequivocally prove the concept. Toward that end, in a recent pilot study treating RIPTag2 mice with an adenovirus expressing a soluble form of PDGFR, we observed pericyte detachment from vessels in pancreatic islet tumors but not normal adjacent exocrine pancreas, supporting the hypothesis that PDGFR signaling is critical for pericyteendothelial association in tumors and is a basis for the effects seen with SU6668 and Gleevec (G. Bergers, unpublished observation).

Not only is the objective response seen with the regimen of Gleevec plus SU5416 conceptually significant, it could be important clinically. Gleevec has been approved for clinical practice by the FDA, and thus it is applicable to a standard protocol for testing investigational drugs, namely combining such candidates with an approved drug. We suggest that Gleevec will show efficacy against human tumors when supplied in combination with an inhibitor of VEGF signaling. Given that a number of drugs aimed at capturing the VEGF-A ligand or inhibiting the VEGFR kinase are in clinical trials, this is a testable hypothesis. Importantly, it should not be necessary that the tumor cells express the kinases that Gleevec targets. Rather, the data presented above suggest that Gleevec (as well as SU6668 and other PDGFR inhibitors) will synergize with inhibitors of VEGF signaling targeting endothelial cells by inhibiting PDGFR signaling in periendothelial cells, thereby targeting interdependent cellular constituents of the tumor vasculature, consequently rendering antiangiogenic therapies more broadly efficacious. This proposition could be tested clinically with Avastin (41) and other inhibitors of 
VEGF signaling in the near term, with the prospect of positively impacting treatment of late-stage disease. In the longer term, a new generation of RTKI with dual specificity against VEGF and PDGFRs (42) have similar potential to significantly impact treatment of wellestablished solid tumors.

\section{Acknowledgments}

We thank Douglas Laird for thoughtful comments on the manuscript, Gerald McMahon and Douglas Laird of Sugen Inc. for providing the SU5416 and SU6668 and for discussions, E. Soliven and J. Imperio for technical assistance, A. McMillan for statistical analysis, and Bill Bowes for support and encouragement. This work was supported by grants from the National Cancer Institute, by the William F. Bowes Foundation, and by start-up funds to G. Bergers from the Department of Neurological Surgery at UCSF.

1. Folkman, J. 2000. Tumor angiogenesis. In Cancer medicine. R.C. Bast, Jr., et al., editors. D.C. Decker Inc. Hamilton, Ontario, Canada. 2546 pp.

2. Kerbel, R., and Folkman, J. 2002. Clinical translation of angiogenesis inhibitors. Nat. Rev. Cancer. 2:727-739.

3. Bergers, G., Javaherian, K., Lo, K.M., Folkman, J., and Hanahan, D. 1999. Effects of angiogenesis inhibitors on multistage carcinogenesis in mice. Science. 284:808-812.

4. Hanahan, D., Christofori, G., Naik, P., and Arbeit, J. 1996. Transgenic mouse models of tumour angiogenesis: the angiogenic switch, its molecular controls, and prospects for preclinical therapeutic models. Eur. J. Cancer. 32A:2386-2393.

5. Folkman, J., Watson, K., Ingber, D., and Hanahan, D. 1989. Induction of angiogenesis during the transition from hyperplasia to neoplasia. Nature. 339:58-61.

6. Hanahan, D., and Folkman, J. 1996. Patterns and emerging mechanisms of the angiogenic switch during tumorigenesis. Cell. 86:353-364.

7. Huss, W., Hanrahan, C., Barrios, R., Simons, J., and Greenberg, N. 2001. Angiogenesis and prostate cancer: identification of a molecular progression switch. Cancer Res. 61:2736-2743.

8. Hanahan, D. 1985. Heritable formation of pancreatic $\beta$-cell tumors in transgenic mice expressing recombinant insulin/simian virus 40 oncogenes. Nature. 315:115-122.

9. Bergers, G., Hanahan, D., and Coussens, L.M. 1998. Angiogenesis and apoptosis are cellular parameters of neoplastic progression in transgenic mouse models of tumorigenesis. Int. J. Dev. Biol. 42:995-1002.

10. Lopez, T., and Hanahan, D. 2002. Elevated levels of IGF-1 receptor convey invasive and metastatic capability in a mouse model of pancreatic islet tumorigenesis. Cancer Cell. 4:339-353.

11. Fong, T.A., et al. 1999. SU5416 is a potent and selective inhibitor of the vascular endothelial growth factor receptor (Flk-1/KDR) that inhibits tyrosine kinase catalysis, tumor vascularization, and growth of multiple tumor types. Cancer Res. 59:99-106.

12. Bergers, G., et al. 2000. Matrix metalloproteinase- 9 triggers the angiogenic switch during carcinogenesis. Nat. Cell Biol. 2:737-744.

13. Bergers, G., and Hanahan, D. 2003. Combining antiangiogenic agents with metronomic chemotherapy enhances efficacy against late-stage pancreatic islet carcinomas in mice. Cold Spring Harb. Symp. Quant. Biol. 67:293-300.

14. Inoue, M., et al. 2002. VEGF-A has a critical non-redundant role in angiogenic switching and pancreatic $\beta$-cell carcinogenesis. Cancer Cell. 1:193-202.

15. Browder, T., et al. 2000. Antiangiogenic scheduling of chemotherapy improves efficacy against experimental drug-resistant cancer. Cancer Res. 60:1878-1886.

16. Klement, G., et al. 2000. Continuous low-dose therapy with vinblastine and VEGF receptor- 2 antibody induces sustained tumor without overt toxicity. J. Clin. Invest. 105:R15-R24.

17. Dash, A.B., et al. 2002. A murine model of CML blast crisis induced by cooperation between BCR/ABL and NUP98/HOXA9. Proc. Natl. Acad. Sci. U. S. A. 99:7622-7627.

18. Parangi, S., Dietrich, W., Christofori, G., Lander, E.S., and Hanahan, D. 1995. Tumor suppressor loci on mouse chromosome 9 and 16 are lost at distinct stages of tumorigenesis in a transgenic model of islet cell carcinoma. Cancer Res. 55:6071-6076.

19. Vajkoczy, P., et al. 1999. Inhibition of tumor growth, angiogenesis, and microcirculation by the novel flk-1 inhibitor SU5416 as assessed by intravital multi-fluorescence videomicroscopy. Neoplasia. 1:31-41.

20. Laird, A.D., et al. 2000. SU6668 is a potent antiangiogenic and antitumor agent that induces regression of established tumors. Cancer Res. 60:4152-4160.

21. Laird, A.D., et al. 2002. SU6668 inhibits Flk-1/KDR and PDGFR $\beta$ in vivo, resulting in rapid apoptosis of tumor vasculature and tumor regression in mice. FASEB J. 16:681-690.

22. Fabbro, D., and Manley, P.W. 2001. Su-6668. SUGEN. Curr. Opin. Investig. Drugs. 2:1142-1148.

23. Solorzano, C.C., et al. 2001. In vivo intracellular signaling as a marker of antiangiogenic activity. Cancer Res. 61:7048-7051.

24. Reinmuth, N., et al. 2001. Induction of VEGF in perivascular cells defines a potential paracrine mechanism for endothelial cell survival. FASEB J. 15:1239-1241.

25. Vajkoczy, P., et al. 2000. Measuring VEGF-Flk-1 activity and consequences of VEGF-Flk-1 targeting in vivo using intravital microscopy: clinical applications. Oncologist. 5(Suppl. 1):16-19.

26. Nehls, V., and Drenckhahn, D. 1993. The versatility of microvascular pericytes: from mesenchyme to smooth muscle? Histochemistry. 99:1-12.

27. Hirschi, K.K., and D'Amore, P.A. 1996. Pericytes in the microvasculature. Cardiovasc. Res. 32:687-698.

28. Morikawa, S., et al. 2002. Abnormalities in pericytes on blood vessels and endothelial sprouts in tumors. Am. J. Pathol. 160:985-1000.

29. Shaheen, R.M., et al. 2001. Tyrosine kinase inhibition of multiple angiogenic growth factor receptors improves survival in mice bearing colon cancer liver metastases by inhibition of endothelial cell survival mechanisms. Cancer Res. 61:1464-1468.

30. Lindahl, P., Johansson, B., Leveen, P., and Betsholtz, C. 1997. Pericyte loss and microaneurysm formation in PDGF-B-deficient mice. Science. 277:242-245.

31. Hellstrom, M., Kaln, M., Lindahl, P., Abramsson, A., and Betsholtz, C. 1999. Role of PDGF-B and PDGFR-beta in recruitment of vascular smooth muscle cells and pericytes during embryonic blood vessel formation in the mouse. Development. 126:3047-3055.

32. Hellstrom, M., et al. 2001. Lack of pericytes leads to endothelial hyperplasia and abnormal vascular morphogenesis. J. Cell Biol. 153:543-553.

33. Compagni, A., Wilgenbus, P., Impagnatelio, M.A., Cotten, M., and Christofori, G. 2000. Fibroblast growth factors are required for efficient tumor angiogenesis. Cancer Res. 60:7163-7169.

34. Buchdunger, E., Matter, A., and Druker, B.J. 2001. Bcr-Abl inhibition as a modality of CML therapeutics. Biochim. Biophys. Acta. 1551:M11-M18.

35. Druker, B.J. 2002. STI571 (Gleevec) as a paradigm for cancer therapy. Trends Mol. Med. 8(Suppl.):S14-S18.

36. Hirschi, K.K., and D'Amore, P.A. 1997. Control of angiogenesis by the pericyte: molecular mechanisms and significance. EXS. 79:419-428.

37. Benjamin, L., Hemo, I., and Keshet, E. 1998. A plasticity window for blood vessel remodelling is defined by pericyte coverage of the preformed endothelial network and is regulated by PDGF-B and VEGF. Development. 125:1591-1598.

38. Pietras, K., et al. 2001. Inhibition of platelet-derived growth factor receptors reduces interstitial hypertension and increases transcapillary transport in tumors. Cancer Res. 61:2929-2934.

39. Sternlicht, M., and Bergers, G. 2000. Matrix metalloproteinases as emerging targets in cancer: status and prospects. ETT. 4:609-633.

40. Coussens, L.M., Fingleton, B., and Matrisian, M. 2002. Matrix metalloproteinase inhibitors and cancer: trials and tribulations. Science. 295:2387-2392.

41. Ferrara, N. 2002. Role of vascular endothelial growth factor in physiologic and pathologic angiogenesis: therapeutic implications. Semin. Oncol. 29:10-14.

42. Laird, A.D., and Cherrington, J.M. 2003. Small molecule tyrosine kinase inhibitors: clinical development of anticancer agents. Expert Opin. Investig. Drugs. 12:51-64.

43. Hanahan, D., and Weinberg, R.A. 2000. The hallmarks of cancer. Cell. 100:57-70. 